Online First version

\title{
Schatten class Toeplitz operators acting on large weighted Bergman spaces
}

\author{
by \\ Hicham Arroussi (Barcelona), Inyoung Park (Pohang) \\ and JORDi PAU (Barcelona)
}

\begin{abstract}
A full description of the membership in the Schatten ideal $S_{p}\left(A_{\omega}^{2}\right)$ for $0<p<\infty$ of Toeplitz operators acting on large weighted Bergman spaces is obtained.
\end{abstract}

1. Introduction. Let $H(\mathbb{D})$ denote the space of all analytic functions on $\mathbb{D}$, where $\mathbb{D}$ is the open unit disk in the complex plane $\mathbb{C}$. A weight is a positive function $\omega \in L^{1}(\mathbb{D}, d A)$, with $d A(z)=\frac{d x d y}{\pi}$ being the normalized area measure on $\mathbb{D}$. The weighted Bergman space $A_{\omega}^{2}$ is the space of all functions $f \in H(\mathbb{D})$ such that

$$
\|f\|_{A_{\omega}^{2}}=\left(\int_{\mathbb{D}}|f(z)|^{2} \omega(z) d A(z)\right)^{1 / 2}<\infty .
$$

We are going to study Toeplitz operators acting on these weighted Bergman spaces, for a certain class $\mathcal{W}$ of radial rapidly decreasing weights. The class $\mathcal{W}$ is the same class of weights considered in [13 and [4, and consists of those radial decreasing weights of the form $\omega(z)=e^{-2 \varphi(z)}$, where $\varphi \in C^{2}(\mathbb{D})$ is a radial function such that $(\Delta \varphi(z))^{-1 / 2} \asymp \tau(z)$ for a radial positive function $\tau(z)$ that decreases to 0 as $|z| \rightarrow 1^{-}$, and $\lim _{r \rightarrow 1^{-}} \tau^{\prime}(r)=0$. Here $\Delta$ denotes the standard Laplace operator. Furthermore, we shall also suppose that either there exists a constant $C>0$ such that $\tau(r)(1-r)^{-C}$ increases as $r \nearrow 1$, or

$$
\lim _{r \rightarrow 1^{-}} \tau^{\prime}(r) \log \frac{1}{\tau(r)}=0
$$

2010 Mathematics Subject Classification: Primary 30H20, 47B35; Secondary 46E15, 47B10. Key words and phrases: weighted Bergman spaces, Toeplitz operators, reproducing kernels, Schatten classes.

Received 8 July 2015; revised 25 November 2015.

Published online *. 
The prototype is the exponential type weight

$$
\omega_{\alpha}(z)=\exp \left(\frac{-1}{\left(1-|z|^{2}\right)^{\alpha}}\right), \quad \alpha>0,
$$

but the class $\mathcal{W}$ also contains the double exponential weights

$$
\omega(z)=\exp \left(-\gamma \exp \left(\frac{\beta}{(1-|z|)^{\alpha}}\right)\right), \quad \alpha, \beta, \gamma>0 .
$$

For weights $\omega$ in our class, the point evaluations $L_{z}$ are bounded linear functionals on $A_{\omega}^{2}$ for each $z \in \mathbb{D}$. In particular, the space $A_{\omega}^{2}$ is a reproducing kernel Hilbert space: for each $z \in \mathbb{D}$, there are functions $K_{z} \in A_{\omega}^{2}$ with $\left\|L_{z}\right\|=\left\|K_{z}\right\|_{A_{\omega}^{2}}$ such that $L_{z} f=f(z)=\left\langle f, K_{z}\right\rangle_{\omega}$, where

$$
\langle f, g\rangle_{\omega}=\int_{\mathbb{D}} f(z) \overline{g(z)} \omega(z) d A(z)
$$

is the natural inner product in $L^{2}(\mathbb{D}, \omega d A)$. The function $K_{z}$ is called the reproducing kernel for the Bergman space $A_{\omega}^{2}$ and has the property that $K_{z}(\xi)=\overline{K_{\xi}(z)}$. The properties of Bergman spaces with exponential type weights have attracted a lot of attention in recent years [3, 4, 6, 7, 13, 14, and new techniques different from the ones used on standard Bergman spaces are required.

Let $\omega \in \mathcal{W}$ and $\mu$ be a finite positive Borel measure on $\mathbb{D}$. The Toeplitz operator $T_{\mu}^{\omega}$ with symbol $\mu$ is given by

$$
T_{\mu} f(z)=T_{\mu}^{\omega} f(z):=\int_{\mathbb{D}} f(\xi) \overline{K_{z}(\xi)} \omega(\xi) d \mu(\xi), \quad f \in H(\mathbb{D}) .
$$

Toeplitz operators acting on various spaces of holomorphic functions have been extensively studied [2, 5, 9, 11, 15, 17], and the theory is especially well understood in the case of Hardy spaces or standard Bergman spaces (see [18] and the references therein). Luecking [9] was the first to study Toeplitz operators on Bergman spaces with measures as symbols, and the study of Toeplitz operators acting on large weighted Bergman spaces was initiated by Lin and Rochberg [8], who proved the following result (as the conditions on the weights are slightly different, we offer a proof in Section 3).

TheOREM 1.1. Let $\omega \in \mathcal{W}$, and $\mu$ be a finite positive Borel measure on $\mathbb{D}$. Then

(i) $T_{\mu}: A_{\omega}^{2} \rightarrow A_{\omega}^{2}$ is bounded if and only if for each $\delta>0$ small enough,

$$
C_{\mu}:=\sup _{z \in \mathbb{D}} \widehat{\mu}_{\delta}(z)<\infty .
$$

Moreover, in that case, $\left\|T_{\mu}\right\| \asymp C_{\mu}$. 
(ii) $T_{\mu}: A_{\omega}^{2} \rightarrow A_{\omega}^{2}$ is compact if and only if for each $\delta>0$ small enough,

$$
\lim _{r \rightarrow 1^{-}} \sup _{|z|>r} \widehat{\mu}_{\delta}(z)=0 .
$$

Here $\widehat{\mu}_{\delta}$ is the averaging function defined as

$$
\widehat{\mu}_{\delta}(z)=\frac{\mu(D(\delta \tau(z)))}{\tau(z)^{2}}, \quad z \in \mathbb{D},
$$

where $\tau(z)$ is the function associated to the weight $\omega$, and $D(\delta \tau(z))$ denotes the euclidian disk centered at $z$ and of radius $\delta \tau(z)$.

Before introducing our main result, we need to review some definitions and properties of Schatten ideals. Let $H$ be a separable Hilbert space, and $0<p<\infty$. The Schatten class $S_{p}=S_{p}(H)$ consists of those compact operators $T$ on $H$ for which the sequence $\left\{\lambda_{n}\right\}$ of singular numbers of $T$ belongs to the sequence space $\ell^{p}$ (the singular numbers are the square roots of the eigenvalues of the positive operator $T^{*} T$, where $T^{*}$ is the Hilbert adjoint of $T$ ). For $p \geq 1$, the class $S_{p}$ is a Banach space with the norm $\|T\|_{p}=\left(\sum_{n}\left|\lambda_{n}\right|^{p}\right)^{1 / p}$, while for $0<p<1$ one has [10] the inequality $\|S+T\|_{p}^{p} \leq\|S\|_{p}^{p}+\|T\|_{p}^{p}$. Also, $T \in S_{p}$ if and only if $T^{*} T \in S_{p / 2}$. We refer to [18, Chapter 1] for a brief account on Schatten classes.

Concerning membership in Schatten ideals of Toeplitz operators, a description for the standard Bergman spaces was obtained by Luecking [9]. For the case of our large Bergman spaces, the following characterization is the main result of this paper, and solves a problem posed by Lin and Rochberg [8].

TheOREM 1.2. Let $\omega \in \mathcal{W}$, and $\mu$ be a finite positive Borel measure on $\mathbb{D}$. For $0<p<\infty$, the Toeplitz operator $T_{\mu}$ is in the Schatten class $S_{p}\left(A_{\omega}^{2}\right)$ if and only if the averaging function $\widehat{\mu}_{\delta}$ is in $L^{p}\left(\mathbb{D}, d \lambda_{\tau}\right)$, where $d \lambda_{\tau}=\tau(z)^{-2} d A(z)$.

Throughout this work, the letter $C$ will denote an absolute constant whose value may change at different occurrences. We also use the notation $a \lesssim b$ to indicate that there is a constant $C>0$ with $a \leq C b$, and the notation $a \asymp b$ means that $a \lesssim b$ and $b \lesssim a$.

The paper is organized as follows. In Section 2 we recall some notation and preliminary results to be used later. We prove Theorem 1.1 on the boundedness and compactness of Toeplitz operators in Section 3, and finally in Section 4 we describe the membership of the Toeplitz operator in the Schatten ideals $S_{p}\left(A_{\omega}^{2}\right)$.

2. Preliminaries and basic properties. A positive function $\tau$ on $\mathbb{D}$ is said to be in the class $\mathcal{L}$ if satisfies the following two properties:

(A) There is a constant $c_{1}$ such that $\tau(z) \leq c_{1}(1-|z|)$ for all $z \in \mathbb{D}$. 
(B) There is a constant $c_{2}$ such that $|\tau(z)-\tau(\zeta)| \leq c_{2}|z-\zeta|$ for all $z, \zeta \in \mathbb{D}$.

We also use the notation

$$
m_{\tau}:=\min \left(1, c_{1}^{-1}, c_{2}^{-1}\right) / 4,
$$

where $c_{1}$ and $c_{2}$ are the constants appearing in the previous definition. For $a \in \mathbb{D}$ and $\delta>0$, we use $D(\delta \tau(a))$ to denote the euclidian disc centered at $a$ and of radius $\delta \tau(a)$. It is easy to see from conditions (A) and (B) (see [13, Lemma 2.1]) that if $\tau \in \mathcal{L}$ and $z \in D(\delta \tau(a))$, then

$$
\frac{1}{2} \tau(a) \leq \tau(z) \leq 2 \tau(a)
$$

for sufficiently small $\delta>0$, namely for $\delta \in\left(0, m_{\tau}\right)$. This fact will be used many times in this work.

Definition 2.1. We say that a weight $\omega$ is in the class $\mathcal{L}^{*}$ if it is of the form $\omega=e^{-2 \varphi}$, where $\varphi \in C^{2}(\mathbb{D})$ with $\Delta \varphi>0$, and $(\Delta \varphi(z))^{-1 / 2} \asymp \tau(z)$, with $\tau(z)$ being a function in the class $\mathcal{L}$. Here $\Delta$ denotes the classical Laplace operator.

It is straightforward to see that $\mathcal{W} \subset \mathcal{L}^{*}$. The following result is from [13. Lemma 2.2] and gives the boundedness of point evaluation functionals on $A_{\omega}^{2}$.

Lemma A. Let $\omega \in \mathcal{L}^{*}, 0<p<\infty, z \in \mathbb{D}$ and $\beta \in \mathbb{R}$. Then there exists $M \geq 1$ such that

$$
|f(z)|^{p} \omega(z)^{\beta} \leq \frac{M}{\delta^{2} \tau(z)^{2}} \int_{D(\delta \tau(z))}|f(\xi)|^{p} \omega(\xi)^{\beta} d A(\xi)
$$

for all $f \in H(\mathbb{D})$ and all $\delta>0$ sufficiently small.

The following lemma on coverings is due to Oleĭnik [12].

Lemma B. Let $\tau$ be a positive function in $\mathbb{D}$ in the class $\mathcal{L}$, and let $\delta \in\left(0, m_{\tau}\right)$. Then there exists a sequence $\left\{z_{j}\right\} \subset \mathbb{D}$ such that

(i) $z_{j} \notin D\left(\delta \tau\left(z_{k}\right)\right), j \neq k$.

(ii) $\bigcup_{j} D\left(\delta \tau\left(z_{j}\right)\right)=\mathbb{D}$.

(iii) $\tilde{D}\left(\delta \tau\left(z_{j}\right)\right) \subset D\left(3 \delta \tau\left(z_{j}\right)\right)$, where $\tilde{D}\left(\delta \tau\left(z_{j}\right)\right)=\bigcup_{z \in D\left(\delta \tau\left(z_{j}\right)\right)} D(\delta \tau(z))$, $j=1,2, \ldots$.

(iv) $\left\{D\left(3 \delta \tau\left(z_{j}\right)\right)\right\}$ is a covering of $\mathbb{D}$ of finite multiplicity $N$.

The multiplicity $N$ in the previous lemma is independent of $\delta$, and it is easy to see that one can take, for example, $N=256$. Any sequence satisfying the conditions in Lemma $\mathrm{B}$ will be called a $(\delta, \tau)$-lattice. 
2.1. Reproducing kernel estimates and test functions. The next result (see [1, 7, 13] for (a), and [8, Lemma 3.6] for (b)) provides useful estimates involving reproducing kernels.

TheOREM A. Let $K_{z}$ be the reproducing kernel of $A_{\omega}^{2}$ where $\omega$ is a weight in the class $\mathcal{W}$. Then:

(a) For each $z \in \mathbb{D}$,

$$
\left\|K_{z}\right\|_{A_{\omega}^{2}}^{2} \omega(z) \asymp \frac{1}{\tau(z)^{2}}, \quad z \in \mathbb{D} .
$$

(b) For all $\delta \in\left(0, m_{\tau}\right)$ sufficiently small,

$$
\left|K_{z}(\zeta)\right| \asymp\left\|K_{z}\right\|_{A_{\omega}^{2}} \cdot\left\|K_{\zeta}\right\|_{A_{\omega}^{2}}, \quad \zeta \in D(\delta \tau(z)) .
$$

The following result on test functions obtained in [13] is basic for our characterization of Schatten class Toeplitz operators.

Lemma C. Let $N \in \mathbb{N} \backslash\{0\}$ and $\omega \in \mathcal{W}$. Then there is a $\rho_{0} \in(0,1)$ such that for each $a \in \mathbb{D}$ with $|a| \geq \rho_{0}$, there is a function $F_{a, N}$ analytic in $\mathbb{D}$ with

$$
\left|F_{a, N}(z)\right|^{2} \omega(z) \asymp 1 \quad \text { if }|z-a|<\tau(a),
$$

and

$$
\left|F_{a, N}(z)\right| \omega(z)^{1 / 2} \lesssim \min \left(1, \frac{\min (\tau(a), \tau(z))}{|z-a|}\right)^{3 N}, \quad z \in \mathbb{D} .
$$

Moreover, the function $F_{a, N}$ belongs to $A_{\omega}^{2}$ with

$$
\left\|F_{a, N}\right\|_{A_{\omega}^{2}} \asymp \tau(a), \quad \rho_{0} \leq|a|<1 .
$$

2.2. Carleson type measures. Let $\mu$ be a finite positive Borel measure on $\mathbb{D}$. We say that $\mu$ is a Carleson measure for $A_{\omega}^{2}$ if there exists a finite positive constant $C$ such that

$$
\int_{\mathbb{D}}|f(z)|^{2} d \mu(z) \leq C\|f\|_{A_{\omega}^{2}}^{2}
$$

for all $f \in A_{\omega}^{2}$. Thus, $\mu$ is Carleson for $A_{\omega}^{2}$ when the inclusion $I_{\mu}: A_{\omega}^{2} \rightarrow$ $L^{2}(\mathbb{D}, \mu)$ is bounded. The next result was proved in [13].

Theorem B. Let $\omega \in \mathcal{W}$, and $\mu$ be a finite positive Borel measure on $\mathbb{D}$. Then:

(a) $I_{\mu}: A_{\omega}^{2} \rightarrow L^{2}(\mathbb{D}, \mu)$ is bounded if and only if for each sufficiently small $\delta>0$

$$
K_{\mu, \omega}:=\sup _{a \in \mathbb{D}} \frac{1}{\tau(a)^{2}} \int_{D(\delta \tau(a))} \omega(\xi)^{-1} d \mu(\xi)<\infty .
$$

Moreover, in that case, $K_{\mu, \omega} \asymp\left\|I_{\mu}\right\|_{A_{\omega}^{2} \rightarrow L^{2}(\mathbb{D}, \mu)}^{2}$. 
(b) $I_{\mu}: A_{\omega}^{2} \rightarrow L^{2}(\mathbb{D}, \mu)$ is compact if and only if for each sufficiently small $\delta>0$,

$$
\lim _{r \rightarrow 1^{-}} \sup _{|a|>r} \frac{1}{\tau(a)^{2}} \int_{D(\delta \tau(a))} \omega(\xi)^{-1} d \mu(\xi)=0 .
$$

Lemma 2.2. Let $\omega \in \mathcal{W}$, and assume that $\widehat{\mu}_{\delta}$ is in $L^{\infty}(\mathbb{D})$ for some small $\delta>0$. Then

$$
\left\langle T_{\mu} f, g\right\rangle_{\omega}=\int_{\mathbb{D}} f(\zeta) \overline{g(\zeta)} \omega(\zeta) d \mu(\zeta), \quad f, g \in A_{\omega}^{2}
$$

Proof. Since $\omega$ is a radial weight, the polynomials are dense in $A_{\omega}^{2}$ and we may assume that $g$ is a holomorphic polynomial. Because of Theorem B, the assumption implies that $d \nu=\omega d \mu$ is a Carleson measure for $A_{\omega}^{2}$. Then

$$
\begin{aligned}
& \int_{\mathbb{D}}\left(\int_{\mathbb{D}}|f(\zeta)|\left|K_{z}(\zeta)\right| \omega(\zeta) d \mu(\zeta)\right)|g(z)| \omega(z) d A(z) \\
& \leq\|f\|_{L^{2}(\nu)} \int_{\mathbb{D}}\left\|K_{z}\right\|_{L^{2}(\nu)}|g(z)| \omega(z) d A(z) \\
& \quad \lesssim\|f\|_{A_{\omega}^{2}} \cdot\|g\|_{\infty} \int_{\mathbb{D}}\left\|K_{z}\right\|_{A_{\omega}^{2}} \omega(z) d A(z) \\
& \lesssim\|f\|_{A_{\omega}^{2}} \cdot\|g\|_{\infty} \int_{\mathbb{D}} \frac{\omega(z)^{1 / 2}}{\tau(z)} d A(z),
\end{aligned}
$$

and this is finite (see [13, Lemma 2.3] for example). Thus, Fubini's theorem gives

$$
\begin{aligned}
\left\langle T_{\mu} f, g\right\rangle_{\omega} & =\int_{\mathbb{D}}\left(\int_{\mathbb{D}} f(\zeta) \overline{K_{z}(\zeta)} \omega(\zeta) d \mu(\zeta)\right) \overline{g(z)} \omega(z) d A(z) \\
& =\int_{\mathbb{D}} f(\zeta)\left(\int_{\mathbb{D}} \overline{g(z)} K_{\zeta}(z) \omega(z) d A(z)\right) \omega(\zeta) d \mu(\zeta) \\
& =\int_{\mathbb{D}} f(\zeta) \overline{\left\langle g, K_{\zeta}\right\rangle_{\omega}} \omega(\zeta) d \mu(\zeta)=\int_{\mathbb{D}} f(\zeta) \overline{g(\zeta)} \omega(\zeta) d \mu(\zeta)
\end{aligned}
$$

3. Proof of Theorem 1.1. Recall that, for $\delta \in\left(0, m_{\tau}\right)$, the averaging function $\widehat{\mu}_{\delta}$ is defined on $\mathbb{D}$ by

$$
\widehat{\mu}_{\delta}(z):=\frac{\mu(D(\delta \tau(z)))}{\tau(z)^{2}}, \quad z \in \mathbb{D} .
$$

3.1. Boundedness. Assume first that $T_{\mu}$ is bounded on $A_{\omega}^{2}$. For fixed $a \in \mathbb{D}$, one has

$$
T_{\mu} K_{a}(a)=\int_{\mathbb{D}}\left|K_{a}(z)\right|^{2} \omega(z) d \mu(z)
$$


By 2.3$)$, there is $\delta \in\left(0, m_{\tau}\right)$ such that $\left|K_{a}(z)\right| \asymp\left\|K_{z}\right\|_{A_{\omega}^{2}} \cdot\left\|K_{a}\right\|_{A_{\omega \omega}^{2}}$ for every $z \in \bar{D}(\delta \tau(a))$. This together with the norm estimate given in (2.2) and the fact that $\tau(z) \asymp \tau(a)$ for $z \in D(\delta \tau(a))$ gives

$$
\begin{aligned}
T_{\mu} K_{a}(a) & \geq \int_{D(\delta \tau(a))}\left|K_{a}(z)\right|^{2} \omega(z) d \mu(z) \\
& \gtrsim \int_{D(\delta \tau(a))}\left\|K_{z}\right\|_{A_{\omega}^{2}}^{2}\left\|K_{a}\right\|_{A_{\omega}^{2}}^{2} \omega(z) d \mu(z) \\
& \asymp \frac{\mu(D(\delta \tau(a)))}{\omega(a) \tau(a)^{4}}=\frac{\widehat{\mu}_{\delta}(a)}{\omega(a) \tau(a)^{2}} .
\end{aligned}
$$

Therefore, by Lemma $\mathrm{A}$ and the estimate of the norm of the reproducing kernels, we obtain

$$
\begin{aligned}
\widehat{\mu}_{\delta}(a) & \lesssim \omega(a) \tau(a)^{2}\left|T_{\mu} K_{a}(a)\right| \leq \omega(a)^{1 / 2} \tau(a)\left\|T_{\mu} K_{a}\right\|_{A_{\omega}^{2}} \\
& \leq \omega(a)^{1 / 2} \tau(a)\left\|T_{\mu}\right\| \cdot\left\|K_{a}\right\|_{A_{\omega}^{2}} \lesssim\left\|T_{\mu}\right\| .
\end{aligned}
$$

Conversely, suppose that (1.1) holds. Let $f, g \in A_{\omega}^{2}$. By Lemma 2.2 and because $d \nu=\omega d \mu$ is a Carleson measure for $A_{\omega}^{2}$ with $\left\|I_{\nu}\right\|^{2} \asymp C_{\mu}:=$ $\sup _{z \in \mathbb{D}} \widehat{\mu}_{\delta}(z)$ (see Theorem $\mathrm{B}$ ), we have

$$
\left|\left\langle T_{\mu} f, g\right\rangle_{\omega}\right| \leq \int_{\mathbb{D}}|f(z)||g(z)| d \nu(z) \leq\|f\|_{L^{2}(\nu)} \cdot\|g\|_{L^{2}(\nu)} \lesssim C_{\mu}\|f\|_{A_{\omega}^{2}} \cdot\|g\|_{A_{\omega}^{2}} .
$$

This shows that $T_{\mu}$ is bounded on $A_{\omega}^{2}$ with $\left\|T_{\mu}\right\| \lesssim C_{\mu}$ finishing the proof.

3.2. Compactness. Let $k_{z}$ be the normalized reproducing kernel in $A_{\omega}^{2}$. From 3.1 in the proof of the boundedness part, and the estimate for $\left\|K_{z}\right\|_{A_{\omega}^{2}}$, we have

$$
\widehat{\mu}_{\delta}(z) \lesssim\left\|T_{\mu} k_{z}\right\|_{A_{\omega}^{2}} .
$$

From Lemma A, it is easy to see that $k_{z}$ converges to zero weakly as $|z| \rightarrow 1^{-}$. Thus, if $T_{\mu}$ is compact, from [18, Theorem 1.14] we obtain (1.2).

Conversely, assume that 1.2 holds, and let $\left\{f_{n}\right\}$ be a sequence in $A_{\omega}^{2}$ converging to zero weakly. To prove compactness, we must show that $\left\|T_{\mu} f_{n}\right\|_{A_{\omega}^{2}} \rightarrow 0$. By the proof of the boundedness, we have

$$
\left\|T_{\mu} f_{n}\right\|_{A_{\omega}^{2}} \lesssim\left\|f_{n}\right\|_{L^{2}(\nu)}
$$

with $d \nu:=\omega d \mu$. By Theorem B, our assumption 1.2 implies that the embedding $I_{\nu}: A_{\omega}^{2} \rightarrow L^{2}(\mathbb{D}, d \nu)$ is compact, which implies that $\left\|f_{n}\right\|_{L^{2}(\nu)}$ tends to zero. Hence $\left\|T_{\mu} f_{n}\right\|_{A_{\omega}^{2}} \rightarrow 0$, proving that $T_{\mu}$ is compact.

4. Membership in Schatten classes. In this section, we are going to describe those positive Borel measures $\mu$ for which the Toeplitz operator $T_{\mu}$ belongs to the Schatten ideal $S_{p}\left(A_{\omega}^{2}\right)$ for $\omega \in \mathcal{W}$. In order to obtain such a characterization, we need to introduce some concepts. 
We define the $\omega$-Berezin transform $B_{\omega} \mu$ of the measure $\mu$ as

$$
B_{\omega} \mu(z):=\int_{\mathbb{D}}\left|k_{z}(\xi)\right|^{2} \omega(\xi) d \mu(\xi), \quad z \in \mathbb{D},
$$

where $k_{z}$ is the normalized reproducing kernel in $A_{\omega}^{2}$. We also consider the measure $\lambda_{\tau}$ given by

$$
d \lambda_{\tau}(z)=\frac{d A(z)}{\tau(z)^{2}}, \quad z \in \mathbb{D} .
$$

Proposition 4.1. Let $1 \leq p<\infty$ and $\omega \in \mathcal{W}$. The following conditions are equivalent:

(a) The function $B_{\omega} \mu$ is in $L^{p}\left(\mathbb{D}, d \lambda_{\tau}\right)$.

(b) The function $\widehat{\mu}_{\delta}$ is in $L^{p}\left(\mathbb{D}, d \lambda_{\tau}\right)$ for any $\delta \in\left(0, m_{\tau}\right)$ small enough.

(c) The sequence $\left\{\widehat{\mu}_{\delta}\left(z_{n}\right)\right\}$ is in $\ell^{p}$ for any $(\delta, \tau)$-lattice $\left\{z_{n}\right\}$ with $\delta \in$ $\left(0, m_{\tau} / 4\right)$ sufficiently small.

Proof. (a) $\Rightarrow$ (b). By Theorem A, for all $\delta \in\left(0, m_{\tau}\right)$ sufficiently small, one has

$$
\left|K_{z}(\zeta)\right| \asymp\left\|K_{z}\right\|_{A_{\omega}^{2}} \cdot\left\|K_{\zeta}\right\|_{A_{\omega}^{2}}, \quad \zeta \in D(\delta \tau(z)) .
$$

Then

$$
\begin{aligned}
B_{\omega} \mu(z) & =\int_{\mathbb{D}}\left|k_{z}(\zeta)\right|^{2} \omega(\zeta) d \mu(\zeta) \geq\left\|K_{z}\right\|_{A_{\omega}^{2}}^{-2} \int_{D(\delta \tau(z))}\left|K_{z}(\zeta)\right|^{2} \omega(\zeta) d \mu(\zeta) \\
& \asymp \int_{D(\delta \tau(z))}\left\|K_{\zeta}\right\|_{A_{\omega}^{2}}^{2} \omega(\zeta) d \mu(\zeta) \asymp \widehat{\mu}_{\delta}(z) .
\end{aligned}
$$

Since $B_{\omega} \mu$ is in $L^{p}\left(\mathbb{D}, d \lambda_{\tau}\right)$, this gives (b).

(b) $\Rightarrow(\mathrm{c})$. Since $\widehat{\mu}_{\delta}\left(z_{n}\right) \lesssim \widehat{\mu}_{4 \delta}(z)$ for $z \in D\left(\delta \tau\left(z_{n}\right)\right)$, we have

$$
\sum_{n} \widehat{\mu}_{\delta}\left(z_{n}\right)^{p} \lesssim \sum_{n} \int_{D\left(\delta \tau\left(z_{n}\right)\right)} \widehat{\mu}_{4 \delta}(z)^{p} \frac{d A(z)}{\tau(z)^{2}} \lesssim \int_{\mathbb{D}} \widehat{\mu}_{4 \delta}(z)^{p} d \lambda_{\tau}(z) .
$$

(c) $\Rightarrow(\mathrm{a})$. We have

$$
B_{\omega} \mu(z) \leq\left\|K_{z}\right\|_{A_{\omega}^{2}}^{-2} \sum_{n} \int_{D\left(\delta \tau\left(z_{n}\right)\right)}\left|K_{z}(s)\right|^{2} \omega(s) d \mu(s) .
$$

By Lemma A,

$$
\left|K_{z}(s)\right|^{2} \omega(s) \lesssim \frac{1}{\tau(s)^{2}} \int_{D(\delta \tau(s))}\left|K_{z}(\xi)\right|^{2} \omega(\xi) d A(\xi) .
$$

This gives

$$
\int_{D\left(\delta \tau\left(z_{n}\right)\right)}\left|K_{z}(s)\right|^{2} \omega(s) d \mu(s) \lesssim\left(\int_{D\left(3 \delta \tau\left(z_{n}\right)\right)}\left|K_{z}(\xi)\right|^{2} \omega(\xi) d A(\xi)\right) \widehat{\mu}_{\delta}\left(z_{n}\right) .
$$


If $p>1$, by Hölder's inequality,

$$
\begin{aligned}
& \left(\sum_{n} \int_{D\left(\delta \tau\left(z_{n}\right)\right)}\left|K_{z}(s)\right|^{2} \omega(s) d \mu(s)\right)^{p} \\
& \quad \lesssim\left\|K_{z}\right\|_{A_{\omega}^{2}}^{2(p-1)} \sum_{n}\left(\int_{D\left(3 \delta \tau\left(z_{n}\right)\right)}\left|K_{z}(\xi)\right|^{2} \omega(\xi) d A(\xi)\right) \widehat{\mu}_{\delta}\left(z_{n}\right)^{p} .
\end{aligned}
$$

This gives

$$
\begin{aligned}
\int_{\mathbb{D}} B_{\omega} \mu & (z)^{p} d \lambda_{\tau}(z) \\
& \lesssim \sum_{n} \widehat{\mu}_{\delta}\left(z_{n}\right)^{p} \int_{D\left(3 \delta \tau\left(z_{n}\right)\right)}\left(\int_{\mathbb{D}}\left|K_{\xi}(z)\right|^{2}\left\|K_{z}\right\|_{A_{\omega}^{2}}^{-2} d \lambda_{\tau}(z)\right) \omega(\xi) d A(\xi) .
\end{aligned}
$$

Since $\left\|K_{z}\right\|_{A_{\omega}^{2}}^{2} \asymp \tau(z)^{-2} \omega(z)^{-1}$, we have

$$
\int_{\mathbb{D}}\left|K_{\xi}(z)\right|^{2}\left\|K_{z}\right\|_{A_{\omega}^{2}}^{-2} d \lambda_{\tau}(z) \asymp\left\|K_{\xi}\right\|_{A_{\omega}^{2}}^{2} \asymp \tau(\xi)^{-2} \omega(\xi)^{-1} .
$$

Inserting this estimate in the previous inequality, we finally get

$$
\int_{\mathbb{D}} B_{\omega} \mu(z)^{p} d \lambda_{\tau}(z) \lesssim \sum_{n} \widehat{\mu}_{\delta}\left(z_{n}\right)^{p}, \quad p \geq 1 .
$$

This finishes the proof.

REMARK. It should be observed that the equivalence of (b) and (c) in Proposition 4.1 continues to hold for $0<p<1$, as also does the implication $(\mathrm{a}) \Rightarrow(\mathrm{b})$. In order to get equivalence with condition (a) even in the case $0<p<1$, it seems that one needs $L^{p}$-integral estimates for reproducing kernels, not available yet.

The next lemma is the analogue in our setting of a well known result for standard Bergman spaces.

Lemma 4.2. Let $\omega \in \mathcal{W}$, and $T$ be a positive operator on $A_{\omega}^{2}$. Let $\widetilde{T}$ be the Berezin transform of the operator $T$, defined by

$$
\widetilde{T}(z)=\left\langle T k_{z}, k_{z}\right\rangle_{\omega}, \quad z \in \mathbb{D} .
$$

(a) Let $0<p \leq 1$. If $\widetilde{T} \in L^{p}\left(\mathbb{D}, d \lambda_{\tau}\right)$ then $T$ is in $S_{p}$.

(b) Let $p \geq 1$. If $T$ is in $S_{p}$ then $\widetilde{T} \in L^{p}\left(\mathbb{D}, d \lambda_{\tau}\right)$.

Proof. Let $p>0$. The positive operator $T$ is in $S_{p}$ if and only if $T^{p}$ is in the trace class $S_{1}$. Fix an orthonormal basis $\left\{e_{k}\right\}$ of $A_{\omega}^{2}$. Since $T^{p}$ is positive, it belongs to the trace class if and only if $\sum_{k}\left\langle T^{p} e_{k}, e_{k}\right\rangle_{\omega}<\infty$. Let $S=\sqrt{T^{p}}$. Then

$$
\sum_{k}\left\langle T^{p} e_{k}, e_{k}\right\rangle_{\omega}=\sum_{k}\left\|S e_{k}\right\|_{A_{\omega}^{2}}^{2}
$$


Now, by Fubini's theorem and Parseval's identity, we have

$$
\begin{aligned}
\sum_{k}\left\|S e_{k}\right\|_{A_{\omega}^{2}}^{2} & =\sum_{k} \int_{\mathbb{D}}\left|S e_{k}(z)\right|^{2} \omega(z) d A(z)=\sum_{k} \int_{\mathbb{D}}\left|\left\langle S e_{k}, K_{z}\right\rangle_{\omega}\right|^{2} \omega(z) d A(z) \\
& =\int_{\mathbb{D}}\left(\sum_{k}\left|\left\langle e_{k}, S K_{z}\right\rangle_{\omega}\right|^{2}\right) \omega(z) d A(z)=\int_{\mathbb{D}}\left\|S K_{z}\right\|_{A_{\omega}^{2}}^{2} \omega(z) d A(z) \\
& =\int_{\mathbb{D}}\left\langle T^{p} K_{z}, K_{z}\right\rangle_{\omega} \omega(z) d A(z)=\int_{\mathbb{D}}\left\langle T^{p} k_{z}, k_{z}\right\rangle_{\omega}\left\|K_{z}\right\|_{A_{\omega}^{2}}^{2} \omega(z) d A(z) \\
& \asymp \int_{\mathbb{D}}\left\langle T^{p} k_{z}, k_{z}\right\rangle_{\omega} d \lambda_{\tau}(z) .
\end{aligned}
$$

Hence, both (a) and (b) are consequences of the inequalities (see [18, Proposition 1.31])

$$
\left\langle T^{p} k_{z}, k_{z}\right\rangle_{\omega} \leq\left[\left\langle T k_{z}, k_{z}\right\rangle_{\omega}\right]^{p}=[\widetilde{T}(z)]^{p}, \quad 0<p \leq 1,
$$

and

$$
[\widetilde{T}(z)]^{p}=\left[\left\langle T k_{z}, k_{z}\right\rangle_{\omega}\right]^{p} \leq\left\langle T^{p} k_{z}, k_{z}\right\rangle_{\omega}, \quad p \geq 1 .
$$

This finishes the proof of the lemma.

Proposition 4.3. Let $\omega \in \mathcal{W}$. If $0<p \leq 1$ and $B_{\omega} \mu$ is in $L^{p}\left(\mathbb{D}, d \lambda_{\tau}\right)$, then $T_{\mu}$ belongs to $S_{p}\left(A_{\omega}^{2}\right)$. Conversely, if $p \geq 1$ and $T_{\mu}$ is in $S_{p}\left(A_{\omega}^{2}\right)$, then $B_{\omega} \mu \in L^{p}\left(\mathbb{D}, d \lambda_{\tau}\right)$.

Proof. If $B_{\omega} \mu$ is in $L^{p}\left(\mathbb{D}, d \lambda_{\tau}\right)$, then it is easy to see that $T_{\mu}$ is bounded on $A_{\omega}^{2}$ (just use the discrete version in Proposition 4.1 to see that the condition in Theorem 1.1 holds). Therefore, the result is a consequence of Lemma 4.2 since $\widetilde{T}_{\mu}(z)=B_{\omega} \mu(z)$.

Now we are almost ready for the characterization of Schatten class Toeplitz operators, but we still need some technical lemmas on properties of lattices. We use the notation

$$
d_{\tau}(z, \zeta)=\frac{|z-\zeta|}{\min (\tau(z), \tau(\zeta))}, \quad z, \zeta \in \mathbb{D} .
$$

Lemma 4.4. Let $\tau \in \mathcal{L}$, and $\left\{z_{j}\right\}$ be $a(\delta, \tau)$-lattice on $\mathbb{D}$. For each $\zeta \in \mathbb{D}$, the set

$$
D_{m}(\zeta)=\left\{z \in \mathbb{D}: d_{\tau}(z, \zeta)<2^{m} \delta\right\}
$$

contains at most $K$ points of the lattice, where $K$ depends on the positive integer $m$ but not on the point $\zeta$.

Proof. Let $K$ be the number of points of the lattice contained in $D_{m}(\zeta)$. Due to the Lipschitz condition (B), we have

$$
\tau(\zeta) \leq \tau\left(z_{j}\right)+c_{2}\left|\zeta-z_{j}\right| \leq\left(1+c_{2} 2^{m} \delta\right) \tau\left(z_{j}\right)=C_{m} \tau\left(z_{j}\right) .
$$


Then

$$
K \cdot \tau(\zeta)^{2} \leq C_{m}^{2} \sum_{z_{j} \in D_{m}(\zeta)} \tau\left(z_{j}\right)^{2} \lesssim C_{m}^{2} \cdot \text { Area }\left(\bigcup_{z_{j} \in D_{m}(\zeta)} D\left(\frac{\delta}{4} \tau\left(z_{j}\right)\right)\right) .
$$

As before, we also have $\tau\left(z_{j}\right) \leq C_{m} \tau(\zeta)$ if $z_{j} \in D_{m}(\zeta)$. From this we easily see that

$$
D\left(\frac{\delta}{4} \tau\left(z_{j}\right)\right) \subset D\left(c 2^{m} \delta \tau(\zeta)\right)
$$

for some constant $c$. Since the sets $\left\{D\left(\frac{\delta}{4} \tau\left(z_{j}\right)\right)\right\}$ are pairwise disjoint, we have

$$
\bigcup_{z_{j} \in D_{m}(\zeta)} D\left(\frac{\delta}{4} \tau\left(z_{j}\right)\right) \subset D\left(c 2^{m} \delta \tau(\zeta)\right)
$$

Therefore, we get

$$
K \cdot \tau(\zeta)^{2} \leq C_{m}^{2} \cdot \text { Area }\left(D\left(c 2^{m} \delta \tau(\zeta)\right)\right) \lesssim C_{m}^{2} 2^{2 m} \tau(\zeta)^{2},
$$

which implies $K \leq C 2^{4 m}$.

Next, we use the result just proved to decompose any $(\delta, \tau)$-lattice into a finite number of "big" separated subsequences.

Lemma 4.5. Let $\tau \in \mathcal{L}$ and $\delta \in\left(0, m_{\tau}\right)$. Let $m$ be a positive integer. Any $(\delta, \tau)$-lattice $\left\{z_{j}\right\}$ on $\mathbb{D}$ can be partitioned into $M$ subsequences such that if $a_{j}$ and $a_{k}$ are different points in the same subsequence, then $d_{\tau}\left(a_{j}, a_{k}\right) \geq 2^{m} \delta$.

Proof. Let $K$ be the number given by Lemma 4.4. From the lattice $\left\{z_{j}\right\}$ we extract a maximal $\left(2^{m} \delta\right)$-subsequence, that is, we select a point $\xi_{1}$ in our lattice, and then we continue selecting points $\xi_{n}$ so that $d_{\tau}\left(\xi_{n}, \xi\right) \geq 2^{m} \delta$ for all previously selected points $\xi$. We stop once the subsequence is maximal, that is, all the remaining points $x$ of the lattice satisfy $d_{\tau}\left(x, \xi_{x}\right)<2^{m} \delta$ for some $\xi_{x}$ in the subsequence. From the remaining points of the lattice we extract another maximal $\left(2^{m} \delta\right)$-subsequence, and we repeat the process until we get $M=K+1$ maximal $\left(2^{m} \delta\right)$-subsequences. If no point of the lattice is left, we are done. On the other hand, if a point $\zeta$ in the lattice is left, this means that there are $M=K+1$ distinct points $x_{\zeta}$ (at least one for each subsequence) in the lattice with $d_{\tau}\left(\zeta, x_{\zeta}\right)<2^{m} \delta$, contrary to the choice of $K$ from Lemma 4.4 .

Now we are ready for the main result of this section, which characterizes the membership in the Schatten ideals of a Toeplitz operator acting on $A_{\omega}^{2}$.

Theorem 4.6. Let $\omega \in \mathcal{W}, \mu$ be a finite positive Borel measure on $\mathbb{D}$, and $0<p<\infty$. The following conditions are equivalent:

(a) The Toeplitz operator $T_{\mu}$ is in $S_{p}\left(A_{\omega}^{2}\right)$.

(b) The function $\widehat{\mu}_{\delta}$ is in $L^{p}\left(\mathbb{D}, d \lambda_{\tau}\right)$ for $\delta \in\left(0, m_{\tau}\right)$ sufficiently small. 
(c) The sequence $\left\{\widehat{\mu}_{\delta}\left(z_{n}\right)\right\}$ is in $\ell^{p}$ for any $\delta>0$ small enough. Moreover, when $p \geq 1$, the previous conditions are also equivalent to:

(d) The function $B_{\omega} \mu$ is in $L^{p}\left(\mathbb{D}, d \lambda_{\tau}\right)$.

Proof. By Proposition 4.1 and the remark following it, statements (b) and (c) are equivalent, and when $p \geq 1$, they are also equivalent to (d). Hence, according to Proposition 4.3, the result is proved for $p=1$, and we have the implication (a) $\Rightarrow(\mathrm{b})$ for $p>1$. Moreover, the implication (c) $\Rightarrow(\mathrm{a})$ for $0<p<1$ is proved in [8] (the conditions on the weights are slightly different, but the same proof works for our class). Thus, it remains to prove (b) $\Rightarrow$ (a) for $p>1$, and (a) $\Rightarrow$ (c) when $0<p<1$.

Let $1<p<\infty$, and assume that $\widehat{\mu}_{\delta} \in L^{p}\left(\mathbb{D}, d \lambda_{\tau}\right)$ with $\delta \in\left(0, m_{\tau}\right)$ small enough. It is not difficult to see, using the equivalent discrete condition in (c) together with Theorem 1.1, that $T_{\mu}$ must be compact. For any orthonormal set $\left\{e_{n}\right\}$ of $A_{\omega}^{2}$, we have

$$
\sum_{n}\left\langle T_{\mu} e_{n}, e_{n}\right\rangle_{\omega}^{p}=\sum_{n}\left(\int_{\mathbb{D}}\left|e_{n}(z)\right|^{2} \omega(z) d \mu(z)\right)^{p} .
$$

By Lemma A and Fubini's theorem,

$$
\begin{aligned}
\int_{\mathbb{D}}\left|e_{n}(z)\right|^{2} \omega(z) d \mu(z) & \lesssim \int_{\mathbb{D}}\left(\frac{1}{\tau(z)^{2}} \int_{D(\delta \tau(z))}\left|e_{n}(\zeta)\right|^{2} \omega(\zeta) d A(\zeta)\right) d \mu(z) \\
& \lesssim \int_{\mathbb{D}}\left|e_{n}(\zeta)\right|^{2} \omega(\zeta) \widehat{\mu}_{\delta}(\zeta) d A(\zeta) .
\end{aligned}
$$

Since $p>1$ and $\left\|e_{n}\right\|_{A_{\omega}^{2}}=1$, we can apply Hölder's inequality to get

$$
\left(\int_{\mathbb{D}}\left|e_{n}(z)\right|^{2} \omega(z) d \mu(z)\right)^{p} \lesssim \int_{\mathbb{D}}\left|e_{n}(\zeta)\right|^{2} \omega(\zeta) \widehat{\mu}_{\delta}(\zeta)^{p} d A(\zeta) .
$$

Plugging this into 4.1) and taking into account that

$$
\left\|K_{\zeta}\right\|_{A_{\omega}^{2}}^{2} \omega(\zeta) \asymp \tau(\zeta)^{-2},
$$

we see that

$$
\begin{aligned}
\sum_{n}\left\langle T_{\mu} e_{n}, e_{n}\right\rangle_{\omega}^{p} & \lesssim \int_{\mathbb{D}}\left(\sum_{n}\left|e_{n}(\zeta)\right|^{2}\right) \omega(\zeta) \widehat{\mu}_{\delta}(\zeta)^{p} d A(\zeta) \\
& \leq \int_{\mathbb{D}}\left\|K_{\zeta}\right\|_{A_{\omega}^{2}}^{2} \omega(\zeta) \widehat{\mu}_{\delta}(\zeta)^{p} d A(\zeta) \\
& \asymp \int_{\mathbb{D}} \widehat{\mu}_{\delta}(\zeta)^{p} d \lambda_{\tau}(\zeta) .
\end{aligned}
$$

By [18, Theorem 1.27] this proves that $T_{\mu}$ is in $S_{p}$ with $\left\|T_{\mu}\right\|_{S_{p}} \lesssim\left\|\widehat{\mu}_{\delta}\right\|_{L^{p}\left(\mathbb{D}, d \lambda_{\tau}\right)}$.

Next, let $0<p<1$, and suppose that $T_{\mu} \in S_{p}\left(A_{\omega}^{2}\right)$. We will prove that (c) holds. The method of proof has its roots in previous work of S. Semmes 
[16] and D. Luecking [9]. Let $\left\{z_{n}\right\}$ be a $(\delta, \tau)$-lattice on $\mathbb{D}$. We want to show that $\left\{\widehat{\mu}_{\delta}\left(z_{n}\right)\right\}$ is in $\ell^{p}$. To this end, we fix a large positive integer $m \geq 2$ and apply Lemma 4.5 to partition the lattice $\left\{z_{n}\right\}$ into $M$ subsequences such that any two distinct points $a_{j}$ and $a_{k}$ in the same subsequence satisfy $d_{\tau}\left(a_{j}, a_{k}\right) \geq 2^{m} \delta$. Let $\left\{a_{n}\right\}$ be such a subsequence and consider the measure

$$
\nu=\sum_{n} \mu \chi_{n}
$$

where $\chi_{n}$ denotes the characteristic function of $D\left(\delta \tau\left(a_{n}\right)\right)$. Since $m \geq 2$, the disks $D\left(\delta \tau\left(a_{n}\right)\right)$ are pairwise disjoint. Since $T_{\mu}$ is in $S_{p}$ and $0 \leq \nu \leq \mu$, we have $0 \leq T_{\nu} \leq T_{\mu}$, which implies that $T_{\nu}$ is also in $S_{p}$. Moreover, $\left\|T_{\nu}\right\|_{S_{p}} \leq$ $\left\|T_{\mu}\right\|_{S_{p}}$. Fix an orthonormal basis $\left\{e_{n}\right\}$ for $A_{\omega}^{2}$ and define an operator $B$ on $A_{\omega}^{2}$ by

$$
B\left(\sum_{n} \lambda_{n} e_{n}\right)=\sum_{n} \lambda_{n} f_{a_{n}}
$$

where $f_{a_{n}}=F_{a_{n}, N} / \tau\left(a_{n}\right)$ and $F_{a_{n}, N}$ are the functions appearing in Lemma C with $N$ so large that $3 N p-4>2 p$. By [13, Proposition 2], the operator $B$ is bounded. Since $T_{\nu} \in S_{p}$, the operator $T=A^{*} T_{\nu} A$ is also in $S_{p}$, with

$$
\|T\|_{S_{p}} \leq\|B\|^{2} \cdot\left\|T_{\nu}\right\|_{S_{p}} .
$$

We split $T$ as $T=D+E$, where $D$ is the diagonal operator on $A_{\omega}^{2}$ defined by

$$
D f=\sum_{n=1}^{\infty}\left\langle T e_{n}, e_{n}\right\rangle_{\omega}\left\langle f, e_{n}\right\rangle_{\omega} e_{n}, \quad f \in A_{\omega}^{2},
$$

and $E=T-D$. By the triangle inequality,

$$
\|T\|_{S_{p}}^{p} \geq\|D\|_{S_{p}}^{p}-\|E\|_{S_{p}}^{p} .
$$

Since $D$ is a positive diagonal operator, we have

$$
\begin{aligned}
\|D\|_{S_{p}}^{p} & =\sum_{n}\left\langle T e_{n}, e_{n}\right\rangle_{\omega}^{p}=\sum_{n}\left\langle T_{\nu} f_{a_{n}}, f_{a_{n}}\right\rangle_{\omega}^{p} \\
& =\sum_{n}\left(\int_{\mathbb{D}}\left|f_{a_{n}}(z)\right|^{2} \omega(z) d \nu(z)\right)^{p} \\
& \geq \sum_{n}\left(\int_{D\left(\delta \tau\left(a_{n}\right)\right)} \frac{\left|F_{a_{n}, N}(z)\right|^{2}}{\tau\left(a_{n}\right)^{2}} \omega(z) d \mu(z)\right)^{p} .
\end{aligned}
$$

Hence, by Lemma C, there is a positive constant $C_{1}$ such that

$$
\|D\|_{S_{p}}^{p} \geq C_{1} \sum_{n} \widehat{\mu}_{\delta}\left(a_{n}\right)^{p} .
$$

On the other hand, since $0<p<1$, by [18, Proposition 1.29] and Lemma 2.2 
we have

$$
\begin{aligned}
\|E\|_{S_{p}}^{p} & \leq \sum_{n} \sum_{k}\left\langle E e_{n}, e_{k}\right\rangle_{\omega}^{p}=\sum_{n, k: k \neq n}\left\langle T_{\nu} f_{a_{n}}, f_{a_{k}}\right\rangle_{\omega}^{p} \\
& \leq \sum_{n, k: k \neq n}\left(\int_{\mathbb{D}}\left|f_{a_{n}}(\xi)\right|\left|f_{a_{k}}(\xi)\right| \omega(\xi) d \nu(\xi)\right)^{p} \\
& =\sum_{n, k: k \neq n}\left(\sum_{j} \int_{D\left(\delta \tau\left(a_{j}\right)\right)}\left|f_{a_{n}}(\xi)\right|\left|f_{a_{k}}(\xi)\right| \omega(\xi) d \mu(\xi)\right)^{p} .
\end{aligned}
$$

If $n \neq k$, then $d_{\tau}\left(a_{n}, a_{k}\right) \geq 2^{m} \delta$. Thus, for $\xi \in D\left(\delta \tau\left(a_{j}\right)\right)$, it is not difficult to see that either

$$
d_{\tau}\left(\xi, a_{n}\right) \geq 2^{m-2} \delta \quad \text { or } \quad d_{\tau}\left(\xi, a_{k}\right) \geq 2^{m-2} \delta .
$$

Indeed, since $n \neq k$, then either $d_{\tau}\left(a_{n}, a_{j}\right) \geq 2^{m} \delta$ or $d_{\tau}\left(a_{j}, a_{k}\right) \geq 2^{m} \delta$. Suppose that $d_{\tau}\left(a_{n}, a_{j}\right) \geq 2^{m} \delta$. If $d_{\tau}\left(\xi, a_{n}\right)<2^{m-2} \delta$, then

$$
\begin{aligned}
\left|a_{n}-a_{j}\right| & \leq\left|a_{n}-\xi\right|+\left|\xi-a_{j}\right|<2^{m-2} \delta \min \left(\tau\left(a_{n}\right), \tau(\xi)\right)+\delta \tau\left(a_{j}\right) \\
& \leq 2^{m-1} \delta \min \left(\tau\left(a_{n}\right), \tau\left(a_{j}\right)\right)+\delta \tau\left(a_{j}\right) .
\end{aligned}
$$

This directly gives a contradiction if $\min \left(\tau\left(a_{n}\right), \tau\left(a_{j}\right)\right)=\tau\left(a_{j}\right)$. In the case that $\min \left(\tau\left(a_{n}\right), \tau\left(a_{j}\right)\right)=\tau\left(a_{n}\right)$, using the Lipschitz condition (B) we get

$$
\left|a_{n}-a_{j}\right|<2^{m-1} \delta \min \left(\tau\left(a_{n}\right), \tau\left(a_{j}\right)\right)+\delta \tau\left(a_{n}\right)+c_{2} \delta\left|a_{n}-a_{j}\right| .
$$

Since $c_{2} \delta \leq 1 / 4$, and $m \geq 2$, we see that this implies

$$
d_{\tau}\left(a_{n}, a_{j}\right)<\frac{4}{3}\left(2^{m-1}+1\right) \delta \leq 2^{m} \delta .
$$

Thus, without loss of generality, we assume that $d_{\tau}\left(\xi, a_{n}\right) \geq 2^{m-2} \delta$. For any $n$ and $k$ we write

$$
I_{n k}(\mu)=\sum_{j} \int_{D\left(\delta \tau\left(a_{j}\right)\right)}\left|f_{a_{n}}(\xi)\right|\left|f_{a_{k}}(\xi)\right| \omega(\xi) d \mu(\xi) .
$$

With this notation and taking into account (4.4), we have

$$
\|E\|_{S_{p}}^{p} \leq \sum_{n, k: k \neq n} I_{n k}(\mu)^{p}
$$

By Lemma C, we have

$$
\left|F_{a_{n}, N}(\xi)\right| \lesssim d_{\tau}\left(\xi, a_{n}\right)^{-3 N}
$$

Apply this inequality raised to the power $1 / 2$, together with the fact that $d_{\tau}\left(\xi, a_{n}\right) \geq 2^{m-2} \delta$, to get

$$
\left|f_{a_{n}}(\xi)\right|=\frac{\left|F_{a_{n}, N}(\xi)\right|^{1 / 2}}{\tau\left(a_{n}\right)}\left|F_{a_{n}, N}(\xi)\right|^{1 / 2} \lesssim 2^{-3 N m / 2} \frac{\left|f_{a_{n}}(\xi)\right|^{1 / 2}}{\tau\left(a_{n}\right)^{1 / 2}} .
$$


We also have

$$
\left|f_{a_{k}}(\xi)\right| \leq\left|f_{a_{k}}(\xi)\right|^{1 / 2} \cdot\left\|K_{\xi}\right\|_{A_{\omega}^{2}}^{1 / 2}
$$

Inserting (4.6) and (4.7) into the definition of $I_{n k}(\mu)$, and using the norm estimate

$$
\left\|K_{\xi}\right\|_{A_{\omega}^{2}} \asymp \tau(\xi)^{-1} \omega(\xi)^{-1 / 2},
$$

we obtain

$$
I_{n k}(\mu) \lesssim \frac{2^{-3 N m / 2}}{\tau\left(a_{n}\right)^{1 / 2}} \sum_{j} \frac{1}{\tau\left(a_{j}\right)^{1 / 2}} \int_{D\left(\delta \tau\left(a_{j}\right)\right)}\left|f_{a_{n}}(\xi)\right|^{1 / 2}\left|f_{a_{k}}(\xi)\right|^{1 / 2} \omega(\xi)^{1 / 2} d \mu(\xi) .
$$

By LemmaA, for $\xi \in D\left(\delta \tau\left(a_{j}\right)\right)$, one has

$$
\begin{aligned}
\left|f_{a_{n}}(\xi)\right|^{1 / 2} \omega(\xi)^{1 / 4} & \lesssim\left(\frac{1}{\tau(\xi)^{2}} \int_{D(\delta \tau(\xi))}\left|f_{a_{n}}(z)\right|^{p / 2} \omega(z)^{p / 4} d A(z)\right)^{1 / p} \\
& \lesssim \tau\left(a_{j}\right)^{-2 / p} S_{n}\left(a_{j}\right)^{1 / p}
\end{aligned}
$$

with

$$
S_{n}(x)=\int_{D(3 \delta \tau(x))}\left|f_{a_{n}}(z)\right|^{p / 2} \omega(z)^{p / 4} d A(z) .
$$

In the same manner we also have

$$
\left|f_{a_{k}}(\xi)\right|^{1 / 2} \omega(\xi)^{1 / 4} \lesssim \tau\left(a_{j}\right)^{-2 / p} S_{k}\left(a_{j}\right)^{1 / p} .
$$

Therefore, there is a positive constant $C_{2}$ such that

$$
\begin{aligned}
I_{n k}(\mu) & \leq C_{2} \cdot \frac{2^{-3 N m / 2}}{\tau\left(a_{n}\right)^{1 / 2}} \sum_{j} \frac{\tau\left(a_{j}\right)^{-4 / p}}{\tau\left(a_{j}\right)^{1 / 2}} S_{n}\left(a_{j}\right)^{1 / p} \cdot S_{k}\left(a_{j}\right)^{1 / p} \mu\left(D\left(\delta \tau\left(a_{j}\right)\right)\right) \\
& =C_{2} \cdot \frac{2^{-3 N m / 2}}{\tau\left(a_{n}\right)^{1 / 2}} \sum_{j} \tau\left(a_{j}\right)^{3 / 2-4 / p} \cdot S_{n}\left(a_{j}\right)^{1 / p} \cdot S_{k}\left(a_{j}\right)^{1 / p} \cdot \widehat{\mu}_{\delta}\left(a_{j}\right) .
\end{aligned}
$$

Since $0<p<1$ and $2^{-3 N m / 2} \leq 2^{-m}$, we get

$$
I_{n k}(\mu)^{p} \leq C_{2}^{p} \cdot \frac{2^{-m p}}{\tau\left(a_{n}\right)^{p / 2}} \sum_{j} \tau\left(a_{j}\right)^{3 p / 2-4} \cdot S_{n}\left(a_{j}\right) \cdot S_{k}\left(a_{j}\right) \cdot \widehat{\mu}_{\delta}\left(a_{j}\right)^{p} .
$$

Bearing in mind (4.5), this gives

$$
\|E\|_{S_{p}}^{p} \leq C_{2}^{p} \cdot 2^{-m p} \sum_{j} \tau\left(a_{j}\right)^{3 p / 2-4} \widehat{\mu}_{\delta}\left(a_{j}\right)^{p}\left(\sum_{n} \frac{S_{n}\left(a_{j}\right)}{\tau\left(a_{n}\right)^{p / 2}}\right) \cdot\left(\sum_{k} S_{k}\left(a_{j}\right)\right) .
$$


On the other hand, we have

$$
\begin{aligned}
\sum_{k} S_{k}\left(a_{j}\right) & =\sum_{k} \int_{D\left(3 \delta \tau\left(a_{j}\right)\right)}\left|f_{a_{k}}(z)\right|^{p / 2} \omega(z)^{p / 4} d A(z) \\
& =\int_{D\left(3 \delta \tau\left(a_{j}\right)\right)}\left(\sum_{k}\left|F_{a_{k}, N}(z)\right|^{p / 2} \tau\left(a_{k}\right)^{-p / 2}\right) \omega(z)^{p / 4} d A(z) .
\end{aligned}
$$

Now, we claim that

$$
\sum_{k} \tau\left(a_{k}\right)^{-p / 2}\left|F_{a_{k}, N}(z)\right|^{p / 2} \lesssim \tau(z)^{-p / 2} \omega(z)^{-p / 4}
$$

In order to prove this, note first that using the estimate 2.4 in Lemma $\mathrm{C}$, the estimate 2.1 and Lemma $B($ iv), we deduce that

$$
\begin{aligned}
\sum_{\left\{a_{k} \in D\left(\delta_{0} \tau(z)\right)\right\}} \tau\left(a_{k}\right)^{-p / 2}\left|F_{a_{k}, N}(z)\right|^{p / 2} & \\
& \lesssim \omega(z)^{-p / 4} \sum_{\left\{a_{k} \in D\left(\delta_{0} \tau(z)\right)\right\}} \tau\left(a_{k}\right)^{-p / 2} \\
& \lesssim \tau(z)^{-p / 2} \omega(z)^{-p / 4} .
\end{aligned}
$$

On the other hand, an application of 2.5 gives

$$
\begin{aligned}
\sum_{\left\{a_{k} \notin D\left(\delta_{0} \tau(z)\right)\right\}} & \tau\left(a_{k}\right)^{-p / 2}\left|F_{a_{k}, N}(z)\right|^{p / 2} \\
& \lesssim \omega(z)^{-p / 4} \tau(z)^{3 N p / 2-p / 2-2} \sum_{\left\{a_{k} \notin D\left(\delta_{0} \tau(z)\right)\right\}} \frac{\tau\left(a_{k}\right)^{2}}{\left|z-a_{k}\right|^{3 N p / 2}} \\
& =\omega(z)^{-p / 4} \tau(z)^{3 N p / 2-p / 2-2} \sum_{j=0}^{\infty} \sum_{a_{k} \in R_{j}(z)} \frac{\tau\left(a_{k}\right)^{2}}{\left|z-a_{k}\right|^{3 N p / 2}}
\end{aligned}
$$

where

$$
R_{j}(z)=\left\{\zeta \in \mathbb{D}: 2^{j} \delta_{0} \tau(z)<|\zeta-z| \leq 2^{j+1} \delta_{0} \tau(z)\right\}, \quad j=0,1, \ldots
$$

Now observe that, using condition $(\mathrm{B})$ in the definition of the class $\mathcal{L}$, it is easy to see that, for $j=0,1, \ldots$,

$$
D\left(\delta_{0} \tau\left(a_{k}\right)\right) \subset D\left(5 \delta_{0} 2^{j} \tau(z)\right) \quad \text { if } a_{k} \in D\left(2^{j+1} \delta_{0} \tau(z)\right) .
$$

Together with the finite multiplicity of the covering (see Lemma B], this gives

$$
\sum_{a_{k} \in R_{j}(z)} \tau\left(a_{k}\right)^{2} \lesssim m\left(D\left(5 \delta_{0} 2^{j} \tau(z)\right)\right) \lesssim 2^{2 j} \tau(z)^{2}
$$


Therefore, as $3 N p-4>2 p$,

$$
\begin{aligned}
& \sum_{\left\{a_{k} \notin D\left(\delta_{0} \tau(z)\right)\right\}} \tau\left(a_{k}\right)^{-p / 2}\left|F_{a_{k}, N}(z)\right|^{p / 2} \\
& \lesssim \omega(z)^{-p / 4} \tau(z)^{-p / 2-2} \sum_{j=0}^{\infty} 2^{-3 N p j / 2} \sum_{a_{k} \in R_{j}(z)} \tau\left(a_{k}\right)^{2} \\
& \lesssim \omega(z)^{-p / 4} \tau(z)^{-p / 2} \sum_{j=0}^{\infty} 2^{(4-3 N p) j / 2} \\
& \lesssim \omega(z)^{-p / 4} \tau(z)^{-p / 2},
\end{aligned}
$$

which together with 4.11) proves the claim 4.10).

Inserting (4.10) into (4.9) gives

$$
\sum_{k} S_{k}\left(a_{j}\right) \lesssim \tau\left(a_{j}\right)^{2-p / 2}
$$

Similarly,

$$
\sum_{n} \frac{S_{n}\left(a_{j}\right)}{\tau\left(a_{n}\right)^{p / 2}} \lesssim \tau\left(a_{j}\right)^{2-p}
$$

Plugging these estimates into 4.8 we finally get

$$
\|E\|_{S_{p}}^{p} \leq C_{2}^{p} \cdot C_{3} \cdot 2^{-m p} \sum_{j} \widehat{\mu}_{\delta}\left(a_{j}\right)^{p}
$$

for some positive constant $C_{3}$. Combining this with (4.2), (4.3) and choosing $m$ large enough that

$$
C_{2}^{p} \cdot C_{3} \cdot 2^{-m p} \leq C_{1} / 2
$$

we deduce that

$$
\sum_{j} \widehat{\mu}_{\delta}\left(a_{j}\right)^{p} \leq \frac{C_{1}}{2}\|T\|_{S_{p}}^{p} \leq C_{4}\left\|T_{\mu}\right\|_{S_{p}}^{p} .
$$

Since this holds for each of the $M$ subsequences of $\left\{z_{n}\right\}$, we obtain

$$
\sum_{n} \widehat{\mu}_{\delta}\left(z_{n}\right)^{p} \leq C_{4} M\left\|T_{\mu}\right\|_{S_{p}}^{p}
$$

for all locally finite positive Borel measures $\mu$ such that

$$
\sum_{n} \widehat{\mu}_{\delta}\left(z_{n}\right)^{p}<\infty
$$

Finally, an easy approximation argument shows that 4.12 actually holds for all locally finite positive Borel measures $\mu$.

Acknowledgements. The second author was supported by the National Research Foundation of Korea (NRF) grant funded by the Korea government (MSIP) (No. 2011-0030044), while the third author was supported by 
DGICYT grants MTM2011-27932-C02-01 and MTM2014-51834-P (Ministerio de Ciencia y Tecnología/MEC) and 2014SGR289 (Generalitat de Catalunya)

\section{References}

[1] A. Borichev, R. Dhuez and K. Kellay, Sampling and interpolation in large Bergman and Fock spaces, J. Funct. Anal. 242 (2007), 563-606.

[2] H. R. Cho, J. Isralowitz and J.-C. Joo, Toeplitz operators on Fock-Sobolev type spaces, Integral Equations Operator Theory 82 (2015), 1-32.

[3] M. Dostanić, Integration operators on Bergman spaces with exponential weight, Rev. Mat. Iberoamer. 23 (2007), 421-436.

[4] P. Galanopoulos and J. Pau, Hankel operators on large weighted Bergman spaces, Ann. Acad. Sci. Fenn. Math. 37 (2012), 635-648.

[5] Z. Hu and X. Lv, Toeplitz operators from one Fock space to another, Integral Equations Operator Theory 70 (2011), 541-559.

[6] T. Kriete and B. MacCluer, Composition operators on large weighted Bergman spaces, Indiana Univ. Math. J. 41 (1992), 755-788.

[7] P. Lin and R. Rochberg, Hankel operators on the weighted Bergman spaces with exponential type weights, Integral Equations Operator Theory 21 (1995), 460-483.

[8] P. Lin and R. Rochberg, Trace ideal criteria for Toeplitz and Hankel operators on the weighted Bergman spaces with exponential type weights, Pacific J. Math. 173 (1996), 127-146.

[9] D. Luecking, Trace ideal criteria for Toeplitz operators, J. Funct. Anal. 73 (1987), 345-368.

[10] C. A. McCarthy, $C_{p}$, Israel J. Math. 5 (1967), 249-271.

[11] T. Mengestie, On Toeplitz operators between Fock spaces, Integral Equations Operator Theory 78 (2014), 213-224.

[12] V. L. Oleĭnik, Embedding theorems for weighted classes of harmonic and analytic functions, J. Soviet Math. 9 (1978), 228-243.

[13] J. Pau and J. Á. Peláez, Embedding theorems and integration operators on Bergman spaces with rapidly decreasing weights, J. Funct. Anal. 259 (2010), 2727-2756.

[14] J. Pau and J. Á. Peláez, Volterra type operators on Bergman spaces with exponential weights, in: Contemp. Math. 561, Amer. Math. Soc., 2012, 239-252.

[15] R. Rochberg and Z. Wu, Toeplitz operators on Dirichlet spaces, Integral Equations Operator Theory 15 (1992), 325-342.

[16] S. Semmes, Trace ideal criteria for Hankel operators, and applications to Besov spaces, Integral Equations Operator Theory 7 (1984), 241-281.

[17] K. Zhu, Positive Toeplitz operators on weighted Bergman spaces of bounded symmetric domains, J. Operator Theory 20 (1988), 329-357.

[18] K. Zhu, Operator Theory in Function Spaces, 2nd ed., Math. Surveys Monogr. 138, Amer. Math. Soc., Providence, RI, 2007. 
Hicham Arroussi, Jordi Pau

Departament de Matemàtica Aplicada i Analisi

Universitat de Barcelona

Gran Via 585

08007 Barcelona, Spain

E-mail: arroussihicham@yahoo.fr jordi.pau@ub.edu
Inyoung Park

Center for Geometry and its Applications Pohang University of Science and Technology Pohang 790-784, South Korea E-mail: iypark26@postech.ac.kr 